\title{
Identifying Projected Clusters from Gene Expression Profiles
}

\author{
Kevin Y. Yip \\ University of Hong Kong \\ ylyip@csis.hku.hk
}

\author{
David W. Cheung \\ University of Hong Kong \\ dcheung@csis.hku.hk
}

\author{
Michael K. Ng \\ University of Hong Kong \\ mng@maths.hku.hk
}

\author{
Kei-Hoi Cheung \\ Yale University \\ kei.cheung@yale.edu
}

\begin{abstract}
In microarray gene expression data, clusters may hide in subspaces. Traditional clustering algorithms that make use of similarity measurements in the full input space may fail to detect the clusters. In recent years a number of algorithms have been proposed to identify this kind of projected clusters, but many of them rely on some critical parameters whose proper values are hard for users to determine. In this paper a new algorithm that dynamically adjusts its internal thresholds is proposed. It has a low dependency on user parameters while allowing users to input some domain knowledge should they be available. Experimental results show that the algorithm is capable of identifying some interesting projected clusters from real microarray data.
\end{abstract}

\section{Introduction}

Clustering is a popular data mining technique for extracting information from gene expression profiles. A large variety of clustering methods have been used to generate many interesting clusters. Some recent studies include $[8,13,17$, 22]. The goal of these methods is to partition similar objects (samples or genes) into clusters such that intra-cluster similarity is maximized while inter-cluster similarity is minimized. Sample clustering is common in tumor studies for identifying tumor subtypes $[4,12,18]$. Gene clustering has been used to predict groups of genes that have similar functions or are co-regulated $[7,11,14]$. It has also become very popular to cluster both samples and genes individually and visualize the results in a single figure [4]. In this paper, we will use the terms object and dimension to mean a row and a column of a dataset respectively. An object refers to a gene when performing gene clustering, and refers to a sample when performing sample clustering. The opposite holds for a dimension.

All the above cited studies assume object similarity is measured in the input space formed by all the dimensions of a dataset. It has been pointed out that gene expression data may exhibit some checkerboard structures [15, 20], in which each block is defined by a subset of objects and a subset of dimensions where the objects are similar when considering only the dimensions. When all dimensions are considered, the objects may appear to be dissimilar. This may occur when, for example, two genes have similar expression patterns only in a subset of samples where certain regulating factors are present. In the other samples, the two genes may express differently. Each block can be viewed as a cluster of objects "projecting" onto a subspace defined by the corresponding dimensions. This kind of clusters is thus referred to as projected clusters [1].

Given a dataset $D$ with $N$ objects and a set $V$ of $d$ input dimensions, a projected cluster $C_{I}$ contains $N_{I}$ objects and is defined in a $d_{I}$-dimensional subspace formed by the set $V_{I}$ of dimensions, where $V_{I} \subseteq V$. In the subspace, the members of $C_{I}$ are similar to each other according to a similarity function, but dissimilar to other objects not in $C_{I} . d_{I}$ is called the dimensionality of cluster $C_{I}$, which is the size of the set of relevant dimensions $V_{I}$ of the cluster. The complementary set $V-V_{I}$ is called the irrelevant dimensions of the cluster. The members of a cluster are dissimilar in the subspace formed by its irrelevant dimensions. A dimension can be relevant to zero, one, or more clusters. To distinguish the clusters defined based on some domain knowledge and the clusters identified by a clustering algorithm, we will call the former ones the real clusters and their relevant dimensions the real relevant dimensions, while the latter kind of clusters will simply be called the clusters and the identified relevant dimensions the selected dimensions.

Notice that the above definition does not assume any kind of object similarity, although a cluster is most often regarded as a group of objects having a small distance from each other (based on a distance function such as Euclidean distance). This kind of clustering, what we will describe as distance-based, has been successful in many studies on gene expression data analysis. For instance, most of the studies cited above implicitly assume distance-based clustering. In this paper we will also introduce a new algorithm that is distance-based. On the other hand, there are situations where it is more suitable to measure the similarity between two objects by their rise and fall expression patterns $[6,16,24]$. Two objects are similar if they have the same direction of response across the relevant dimensions, regardless of their absolute expression values. We will discuss later how this kind of pattern-based clustering can be handled by a modified distance-based clustering algorithm.

The goal of projected clustering algorithms is to partition the objects into high-quality projected clusters. Basically, a cluster is of high quality if its member objects are unexpectedly similar. A formal quality measure will be described in Section 3. We will first assume clusters are dis- 
joint, i.e., each object belongs to only one cluster, and later extend our study to consider non-disjoint clusters since they are common in gene clustering where each gene may belong to multiple groups according to different categorizations.

In the next section, we will review some projected clustering approaches proposed in recent years, and discuss some of their potential problems. A new algorithm will be proposed in Section 3, which is designed to avoid the problems. Experimental results on real datasets will be presented in Section 4, and some discussions and the conclusion of the study will be given in Section 5 and Section 6 respectively.

\section{Related Work}

There have been a lot of studies on projected clustering and its related problems subspace clustering [3] and biclustering [6] in recent years. A thorough survey of the three problems can be found in [25]. In this section we focus on the related work on the projected clustering problem, which assumes a distance-based similarity definition and produces disjoint clusters. We are especially interested in this problem because of the large number of fruitful studies on clustering gene expression profiles that also make the two assumptions, and the few reported studies on applying projected clustering on gene expression profiles.

There are two major challenges in projected clustering that make it distinctive from traditional clustering. The first challenge is the simultaneous determination of both cluster members and relevant dimensions. Cluster members are determined by calculating object distances in the subspace formed by the relevant dimensions, while the relevant dimensions are determined by measuring the distances between the projections of the cluster members along different dimensions. One common approach to tackling this chicken-and-egg problem is to form some tentative clusters according to some heuristics, determine their relevant dimensions, and then refine the cluster members based on the selected dimensions. The heuristics being used are critical to the effectiveness of the algorithm. If inappropriate heuristics are used, the tentative clusters formed will not help the discovery of real clusters.

The second challenge is determining the dimensionality of each cluster, which is usually unknown to users when working on gene expression profiles due to the lack of domain knowledge and the large number of possible values given the high dimensionality of data.

We now review some proposed projected clustering approaches. The partitional approach PROCLUS [1] is based on the k-medoids method [19]. As in traditional k-medoids methods, some objects are initially chosen as the medoids. But before assigning every object in the dataset to the nearest medoid, each medoid is first temporarily assigned a set of neighboring objects that are close to it in the input space to form a tentative cluster. For each tentative cluster, all dimensions are sorted according to the average distance between the projections of the medoid and the neighboring objects. On average $l$ dimensions with the smallest average distances are selected as the relevant dimensions for each cluster, where $l$ is a user parameter. Normal object assignment then resumes, but the distance between an object and a medoid is computed using only the selected dimensions. Medoids with too few assigned objects are regarded as outliers, which are replaced by some other objects to start a new iteration.

The user parameter $l$ may introduce a usability problem since the correct value to use is hard to determine. Another potential problem arises when the real clusters have few relevant dimensions, in which case the cluster members may not be close to each other in the full input space. Since the tentative clusters are formed based on distance calculations in the input space, when a member of a real cluster is chosen as a medoid, the neighboring objects assigned to it may not come from the same real cluster. Subsequently, the dimensions selected would not be the real relevant dimensions and the resulting cluster would be mixed of objects from different real clusters.

Another partitional algorithm ORCLUS [2] was proposed to improve PROCLUS. According to the experimental results reported in [2], it is more accurate and stable than PROCLUS. Nevertheless, it still relies on user-supplied values in deciding the number of dimensions to select for each cluster.

In the hypercube approach DOC and its variant FastDOC [21], each cluster is defined as a hypercube with width $2 \omega$, where $\omega$ is a user parameter. The clusters are formed one after another. To find a cluster, a pivot point is randomly chosen as the cluster center and a small set of objects is randomly sampled to form a tentative cluster around the pivot point. A dimension is selected if and only if the distance between the projected values of every sample and the pivot point on the dimension is no more than $\omega$. The tentative cluster is thus bounded by a hypercube with width $2 \omega$. All objects in the dataset falling into the hypercube are grouped to form a candidate cluster. More random samples and pivot points are then tried to form more candidate clusters, and a specially designed function is used to evaluate the quality of them. The candidate cluster with the best evaluation score is accepted, and the whole process repeats to find other clusters.

As with PROCLUS and ORCLUS, the selected dimensions of DOC and FastDOC are determined by a user parameter. In addition, they also restrict each cluster to be a hypercube with equal width along all relevant dimensions, which is unlikely to be true in real data. Tentative clusters are formed by random sampling, which avoids direct distance calculations in the input space. However, the number of tentative clusters required to try can become so large that seriously affects the speed performance.

Summarizing the above observations, in order to apply projected clustering on gene expression data, it would be preferable to develop an algorithm that can identify the dimensionalities of the clusters directly from data and avoid the formation of problematic tentative clusters. In the next 
section we will describe a new projected clustering algorithm HARP (a Hierarchical approach with Automatic Relevant dimension selection for Projected clustering) [25] that satisfies these requirements. It is an agglomerative hierarchical clustering algorithm with each object treated as a singleton cluster at the beginning, and the most similar clusters are merged iteratively according to a merge score. The building components of the algorithm will be introduced first, followed by a description of the complete algorithm and some possible extensions.

\section{The HARP Algorithm}

\subsection{Relevance Index, Cluster Quality and Merge Score}

In distance-based projected clustering, a cluster can be viewed as a group of objects being unexpectedly close to each other in a certain subspace. In other words, for a dimension to be relevant to a cluster, the projections of the cluster members on the dimension should be unexpectedly close to each other. This closeness can be measured by the ratio of the variance within the cluster to the variance in the whole dataset. Denote $\sigma_{I j}^{2}$ as the variance of projected values of all objects in $C_{I}$ along dimension $v_{j}$ (the local variance) and $\sigma_{. j}^{2}$ as the variance of projected values along $v_{j}$ in the whole dataset (the global variance), the relevance index of $v_{j}$ in cluster $C_{I}$ is defined as follows:

$$
R_{I j}=1-\frac{\sigma_{I j}^{2}}{\sigma_{\cdot j}^{2}} .
$$

The index gives a high value when the local variance is small compared to the global variance, which refers to the situation where the projections of the cluster members on the dimension are close, and the closeness is not due to a small average distance between the projected values in the whole dataset. A dimension receives an index value close to the maximum of one if the local variance is extremely small, which means the projections form an excellent signature for identifying the cluster members. Alternatively, if the local variance is only as small as the global variance, the dimension will receive an index value of zero. This suggests a baseline for dimension selection: a negative $R$ value indicates a dimension is not more relevant to a cluster than to a random sample of objects. The dimension should therefore not be selected. We will discuss later how this baseline is used to define the stopping criteria of HARP.

Based on the relevance index, the quality of a cluster $C_{I}$ can be measured by the sum of the index values of all the selected dimensions:

$$
Q_{I}=\sum_{v_{j} \in V_{I}} R_{I j}
$$

In general, the more selected dimensions a cluster has and the larger are their respective $R$ values, the larger will be the value of $Q$. We define the quality measure in this way since an identified cluster is more likely to be consist of objects from the same real cluster if the identified cluster has more selected dimensions and the dimensions have higher relevance index values [25]. We will discuss how HARP determines the relevant dimensions of each cluster later. At this point it can be assumed that each cluster has a reasonable set of selected dimensions.

Similarly, a score can be defined to evaluate the merge between two clusters. Basically, if two clusters can be merged to form a cluster with a high quality, the merge is a potentially good one, i.e., the two clusters probably contain objects from the same real cluster. However, in case the two merging clusters have a large size difference, an unfavorable situation called mutual disagreement can occur. Consider a large cluster with a thousand objects and a small one with only five objects. If they are merged to form a new cluster, its mean and variance of projected values will highly resemble the original values of the large cluster, which will dominate the choice of the dimensions to be selected. If a dimension is originally selected by the large cluster, it will probably be selected by the new cluster also no matter the projected values of the small cluster are close to those of the large cluster or not. The resulting cluster can have a high $Q$ score even the two clusters have a strong mutual disagreement on the signatures of the resulting cluster.

To cope with this problem, we modify the relevance index to take into account the mutual disagreement phenomenon. Suppose $C_{I_{3}}$ is the resulting cluster formed by merging $C_{I_{1}}$ and $C_{I_{2}}$, the mutual-disagreement-sensitive relevance index of dimension $v_{j}$ in $C_{I_{3}}$ is defined as follows:

$$
\begin{aligned}
R_{I_{3} j}^{*} & =\frac{R_{I_{1} j \mid I_{2}}+R_{I_{2} j \mid I_{1}}}{2}, \\
R_{I_{1} j \mid I_{2}} & =1-\frac{\sigma_{I_{1} j}^{2}+\left(x_{I_{1} j}-x_{I_{2} j}\right)^{2}}{\sigma_{\cdot j}^{2}} \\
& =1-\frac{\sum_{x_{i} \in C_{I_{1}}}\left(x_{i j}-x_{I_{2} j}\right)^{2} / N_{i}}{\sigma_{\cdot j}^{2}},
\end{aligned}
$$

where $x_{i j}$ is the projection of object $x_{i}$ on dimension $v_{j}$, and $x_{I j}$ is the mean projected value of all members of cluster $C_{I}$ on $v_{j} . R_{I_{1} j \mid I_{2}}$ is the adjusted relevance index of $v_{j}$ in $C_{I_{1}}$ given that $C_{I_{1}}$ is merging with $C_{I_{2}}$. The numerator of its second term is the average squared distance between the projected values of $C_{I_{1}}$ on $v_{j}$ from the mean projected value of $C_{I_{2}} . R_{I_{2} j \mid I_{1}}$ is defined similarly. If the two clusters do not agree on the values along $v_{j},\left(x_{I_{1} j}-x_{I_{2} j}\right)^{2}$ will effectively diminish the $R^{*}$ score of the dimension. The original $R$ index is used to determine the quality of a cluster, while the modified index $R^{*}$ is used to determine the merge score between two clusters. When $C_{I_{1}}$ and $C_{I_{2}}$ are merged to form $C_{I_{3}}$, the merge score is as follows:

$$
\begin{aligned}
= & \sum_{v_{j} \in V_{I_{3}}} R_{I_{3} j}^{*}\left(C_{I_{1}}, C_{I_{2}}\right) \\
= & \sum_{v_{j} \in V_{I_{3}}} \frac{R_{I_{1} j \mid I_{2}}+R_{I_{2} j \mid I_{1}}}{2}
\end{aligned}
$$




$$
=\sum_{v_{j} \in V_{I_{3}}}\left[1-\frac{\sigma_{I_{1} j}^{2}+\sigma_{I_{2}, j}^{2}+2\left(x_{I_{1} j}-x_{I_{2} j}\right)^{2}}{\sigma_{\cdot j}^{2}}\right] .
$$

The $M S$ score will be used to determine the merge order. Merges with higher $M S$ scores will be allowed to perform earlier.

\subsection{Dynamic Threshold Loosening}

When we introduced the $M S$ function, we assumed that there is a way to determine the relevant dimensions of each cluster. In this section we discuss how this is made possible by the dynamic threshold loosening mechanism.

As discussed in Section 3.1, a cluster is more likely to be correct if it contains a larger number of selected dimensions, and the selected dimensions have higher relevance index values. This means merges that form resulting clusters with both properties should be allowed to perform earlier. Practically, this is achieved by two internal thresholds $R_{\min }$ and $d_{\min }$. Two clusters are allowed to merge if and only if the resulting cluster has $d_{\min }$ or more selected dimensions, and a dimension $v_{j}$ is selected by $C_{I}$ if and only if $R_{I j}^{*} \geq R_{\min }$. At any time, the two thresholds define a set of allowed merges where the actual merging order within the set is determined by the $M S$ scores.

At the beginning, $R_{\min }$ and $d_{\min }$ are initialized to their tightest (i.e., highest) values 1 and $d$ respectively. All allowed merges produce clusters that contain identical objects, so the clusters must be correct. At some point, there will be no more qualified merges. The thresholds will be slightly loosened to qualify some new merges. Whenever all qualified merges have been performed, the thresholds will be further loosened. As clustering proceeds, the clusters grow bigger in size. The projections of the cluster members on the real relevant dimensions remain close to each other, but the chance of having similar closeness of projections along other dimensions drops, so as their relevance index values. This allows the real relevant dimensions to be clearly differentiated from the irrelevant ones, which in turn ensures the formation of correct clusters.

In order to guarantee the quality of the final clusters, the two thresholds are associated with baseline values such that when the baselines are reached, no further loosening is allowed. As mentioned in Section 3.1, a negative $R$ value means that a dimension is very unlikely to be relevant to a cluster. The baseline of $R_{\min }$ is thus set to zero. For $d_{\min }$, the baseline is set to one, which is the minimum value for a cluster to be defined as a projected cluster. We will see later that the HARP algorithm allows users to specify an optional target number of clusters. According to our experience, if such a value is specified, the algorithm usually finishes the clustering process well before the thresholds reach their baselines. The clusters produced thus contain selected dimensions with $R$ scores much better than that of a random set of projected values.

There are many possible ways to loosen the threshold values. From our empirical study, a simple linear loosening scheme is found to be very adaptive and performed well. In this scheme, there is a fixed number of threshold levels such that whenever no more qualified merges remain, the values of the two thresholds are updated using a linear interpolation towards the baseline values (see Section 3.3 for details). By default, we set the number of threshold loosening steps to the dataset dimensionality $d$ such that after each threshold loosening, $d_{\min }$ is reduced by 1 .

Obviously, while the simple loosening mechanism and the default number of loosening steps work well in our experiments, they are not always the best choice. To this end, we allow users to input some domain knowledge should they be available. Users are allowed to input the initial and baseline values for the two thresholds and the number of loosening steps. They may also select an alternative loosening scheme (e.g. aggressive loosening that always loosens the threshold that leads to more qualified merges, or conservative loosening that does the reverse), or specify their preferred scheme as a plugin procedure.

\subsection{The Complete Algorithm}

The whole algorithm is shown in Algorithm 1. At the beginning of the clustering process, each object forms a singleton cluster. The dimensionality and relevance thresholds $d_{\min }$ and $R_{\min }$ are initialized to their tightest values. For each cluster, the dimensions that satisfy the threshold requirements are selected. The merge score between each pair of clusters is then calculated. Only merges that form a resulting cluster with $d_{\min }$ or more selected dimensions are qualified and the others are ignored.

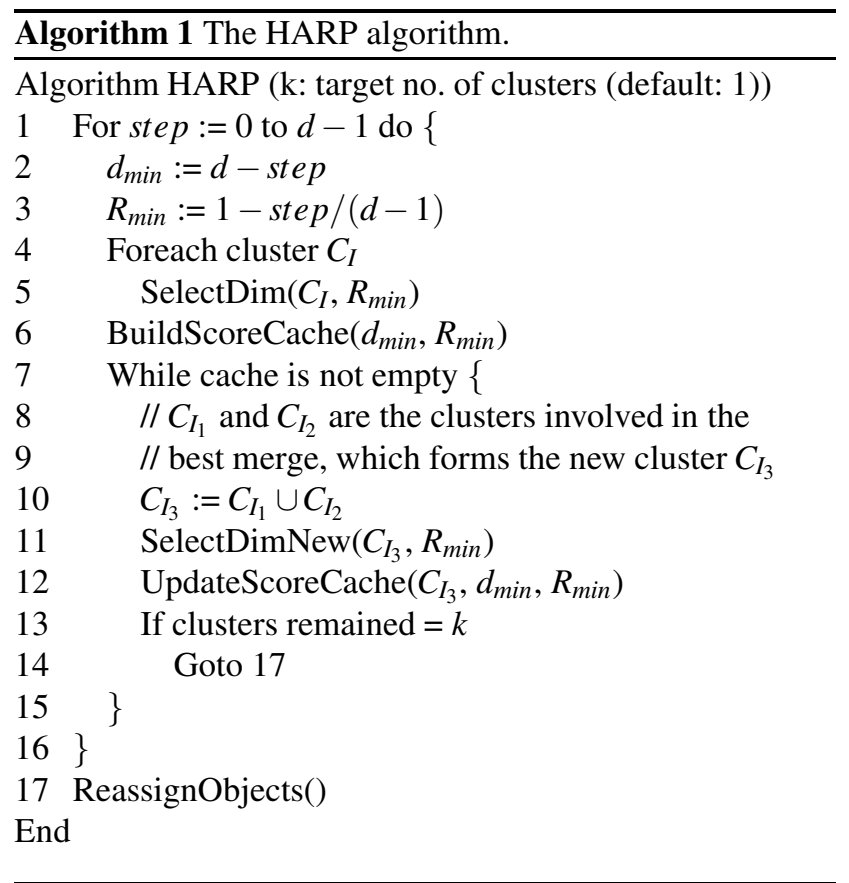

The algorithm repeatedly performs the best merge according to the $M S$ scores of the qualified merges. In order to efficiently determine the next best merge, merge scores 
are stored in a cache (e.g. a quad tree or a Conga line [10]). After each merge, the scores related to the merged clusters are removed from the cache, and the best scores of the qualified merges that involve the new cluster are inserted back. The selected dimensions of the new cluster are determined by its members according to $R_{\min }$. According to the definition of $R$, if a dimension is originally not selected by both merging clusters, it must not be selected by the new cluster. However, if a dimension is originally selected by one or both of the merging clusters, it may or may not be selected by the new cluster.

Whenever the cache becomes empty, there are no more qualified merges at the current threshold level. The thresholds will be loosened linearly according to the formulas in lines 2 and 3 of Algorithm 1. Further rounds of merging and threshold loosening will be carried out until a target number of clusters remain, or the thresholds reach their baseline values and no more qualified merges exist.

To further improve clustering accuracy, an optional object reassignment step can be performed after the completion of the hierarchical part. The $M S$ score between each clustered object and each cluster is computed based on the final threshold values when the hierarchical part ends. After computing all the scores, each of the objects is assigned to the cluster with the highest $M S$ score. The process repeats until convergence or a maximum number of iterations are reached.

The parameter $k$ that specifies the target number of clusters is optional. Like other hierarchical clustering methods, $k$ can be set to 1 and the whole clustering process can be logged as a dendrogram, which allows users to determine the cluster boundaries from a graphical representation (e.g. [9]), or cut the tree according to the merge order of the clusters and a value of $k$ determined a posteriori. Due to the threshold requirements, it is not always possible to merge the objects into a single cluster at the end of clustering. In general, the dendrograms of HARP are forests of trees. Also, it can be observed that the dynamic threshold loosening mechanism relies on the hierarchical nature of HARP. These explain why we adopt the hierarchical approach in spite of its intrinsic high time complexity. HARP is especially suitable for applications where accuracy is the first priority and the datasets are of moderate sizes, such as gene expression profiles. For instance, clustering a typical gene expression dataset with 5000 genes and 50 samples takes ten to twenty minutes on a desktop PC, which is quite reasonable. It is also possible to improve the speed performance of HARP in a number of ways. The details can be found in [25].

\subsection{Extensions}

As discussed previously, there are situations where pattern-based clustering and non-disjoint clusters are desirable. HARP can be extended to satisfy these two requirements. To consider pattern-based similarity, the input dataset is first preprocessed by subtracting each expres- sion value by the row average so that all resulting rows have a zero mean. Each resulting expression value measures the relative expression level of the object on the particular dimension. The distance between two preprocessed objects captures their pattern similarity in the full input space. A similar mechanism is carried out to determine the pattern similarity between two clusters in the subspace of the resulting cluster formed by merging the clusters. Suppose clusters $C_{I_{1}}$ and $C_{I_{2}}$ have relevant dimensions $V_{I_{1}}$ and $V_{I_{2}}$ respectively, and they can be merged to form $C_{I_{3}}$. The potential set of relevant dimensions of $C_{I_{3}}, V_{I_{3}}^{e s t}$, is estimated by the intersection of $V_{I_{1}}$ and $V_{I_{2}}$. Each object in $C_{I_{1}}$ and $C_{I_{2}}$ subtracts their expression values by the mean expression along the dimensions in $V_{I_{3}}^{\text {est }}$. The distance between the two clusters in the subspace formed by $V_{I_{3}}^{\text {est }}$ thus captures their pattern similarity in the subspace. The set of selected dimensions can be refined by comparing the relevance index value of each dimension with the $R_{\min }$ threshold, and the process can be repeated a few times to identify a satisfactory set of selected dimensions.

When clustering completes, for each produced cluster $C_{I}$, all the objects in the dataset will be examined to see if they can be merged into $C_{I}$ without lowering its quality. Each object is regarded as a singleton cluster, and its expression values are adjusted as described above according to the relevant dimensions of $C_{I}$. The $M S$ score between it and $C_{I}$ is calculated subject to the thresholds where $d_{\min }$ and $R_{\min }$ are set as the number and minimum $R$ value of the relevant dimensions of $C_{I}$. All the objects involved in the allowed merges are assigned as members of $C_{I}$. Since each object can be assigned to multiple clusters, the final clusters are likely to be non-disjoint.

\section{Experiments}

In this section we present the experimental results of HARP on two real datasets. Due to space limitation, we omit other extensive experimental results that compare HARP with seven projected and non-projected clustering algorithms on both synthetic and real datasets. The results show that HARP is able to identify some projected clusters hidden in some low-dimensional space that are missed by the other algorithms. The details can be found in [25].

\subsection{Datasets}

Lymphoma: It is a dataset used in studying distinct types of diffuse large B-cell lymphoma (DLBCL)(Figure 1 of [4]). It contains 96 samples, each with 4026 expression values. The samples are categorized into 9 classes according to the category of mRNA sample studied. We used HARP to perform distance-based clustering to produce 9 sample clusters. Each relevant dimension of a cluster represents a gene that has similar expression levels in 
the member samples of the cluster, which is a potential signature of the sample type.

Yeast: The original dataset was published in [7]. It contains the expression levels of 6,218 yeast ORFs at 17 time points taken at 10 minute intervals, which cover nearly two full cell cycles. The dataset used here is the subset selected according to [23] that contains 2,884 genes. We preprocessed the data according to the method suggested in [6], and used HARP to perform pattern-based clustering to produce non-disjoint gene clusters using the two extensions. As in [6], we treated two genes as similar if they have complementary expression patterns in the relevant subspace, i.e., the two genes constantly show opposite rise and fall patterns across the relevant dimensions. This is accomplished by having two copies of each gene in the dataset, one with the original expression values, and the other the negation of them. This results in two nearly identical copies of every cluster being formed. In the results reporting in the coming sections, all duplicated clusters and duplicated genes in a cluster are removed.

\subsection{Results}

Lymphoma: HARP was able to separate the samples of different types to different clusters with only a small number of errors. Some interesting clusters located at the top two levels of the dendrogram are listed in Table 1 . We investigated the importance of dimension selection in the clustering process by calculating the distance ratios $A_{1}$ to $A_{3}$ defined as follows:

$$
\begin{aligned}
& A_{1}\left(C_{I}\right)=\frac{\sum_{x_{i} \in C_{I}, v_{j} \in V_{I}}\left(x_{i j}-x_{I j}\right)^{2} / d_{I}}{\sum_{x_{i} \in C_{I}, v_{j} \in V}\left(x_{i j}-x_{I j}\right)^{2} / d} \\
& A_{2}\left(C_{I}\right)=\frac{\sum_{x_{i} \in C_{I}, v_{j} \notin V_{I}}\left(x_{i j}-x_{I j}\right)^{2} /\left(d-d_{I}\right)}{\sum_{x_{i} \in C_{I}, v_{j} \in V}\left(x_{i j}-x_{I j}\right)^{2} / d} \\
& A_{3}\left(C_{I}\right)=\frac{\sum_{x_{i} \notin C_{I}, v_{j} \in V_{I}}\left(x_{i j}-x_{I j}\right)^{2} / d_{I}}{\sum_{x_{i} \notin C_{I}, v_{j} \in V}\left(x_{i j}-x_{I}\right)^{2} / d}
\end{aligned}
$$

$A_{1}$ measures the increase in compactness of the cluster due to dimension selection, $A_{2}$ measures how irrelevant are the non-selected dimensions, and $A_{3}$ measures the increase in separation between the cluster members and other objects due to the selection. For a good cluster, $A_{1}$ should be smaller than one, $A_{2}$ should be greater than one, and $A_{3}$ should be larger than $A_{1}$. All clusters in Table 1 satisfy these requirements, which means the selection of relevant dimensions makes the cluster members more distinguishable. For each cluster of samples, we also randomly selected 100,000 sets of relevant dimensions and calculated the corresponding distance ratios. All the resulting ratios are very close to one with standard deviations not more than $10^{-5}$, which verify that the relevant dimensions selected by HARP are statistically unexpected and significantly better than random selections.

We then examined the biological meaning of the selected dimensions of the clusters. In Figure 2 of [4], some genes are highlighted as the signatures of some sample types or biological processes: proliferation, germinal centre $\mathrm{B}$, lymph

\begin{tabular}{lcccc}
\hline Samples & $\begin{array}{c}\text { Selected } \\
\text { genes }\end{array}$ & $A_{1}$ & $A_{2}$ & $A_{3}$ \\
\hline 6 RAT & 2456 & 0.72 & 1.32 & 0.87 \\
43 DLBCL, 2 NILNT & 3515 & 0.96 & 1.25 & 1.02 \\
10 ABB, 1 TCL & 2734 & 0.80 & 1.32 & 1.00 \\
9 FL, 2 GCB, 2 RBB & 3104 & 0.85 & 1.38 & 1.00 \\
11 CLL, 2 RBB & 2614 & 0.82 & 1.27 & 0.97 \\
16 DLBCL & 3347 & 0.90 & 1.38 & 1.01 \\
27 DLBCL, 2 NILNT & 3610 & 0.96 & 1.32 & 1.00 \\
\hline
\end{tabular}

Table 1. The distance ratios of some clusters identified by HARP from the lymphoma data.

node and $\mathrm{T}$ cell. For each cluster formed by HARP, we sorted all the genes in descending order according to their $R$ values, and checked the ranks of the signature genes. It was found that the large DLBCL cluster contains many signature genes in the proliferation region receiving high ranks, which suggests that the expression values of the genes could potentially be used to identify DLBCL samples. Similarly, it was found that the resting/activated T samples have a distinctive expression pattern. The 6 samples form a clear cluster with many of the signature genes receiving very large $R$ values. Activated blood $\mathrm{B}, \mathrm{FL}$ and CLL samples formed three separate clusters consisting of few samples from other types. They all have large $R$ values at the signature genes at the lymph node region due to the constantly low expression, but the three types of samples were successfully separated into different clusters according to the expression values of other relevant genes, in particular those in the germinal centre $\mathrm{B}$ region.

Yeast: We used HARP to produce about 100 distinct clusters and compared them with the 100 biclusters reported in [6]. Table 2 compares some statistics of the two sets of clusters. The $H$ score of a cluster is the average squared residue score defined as follows:

$$
H_{I}=\frac{\sum_{x_{i} \in C_{I}, v_{j} \in V_{I}}\left(x_{i j}-x_{I j}-x_{i J}+x_{I J}\right)^{2}}{N_{I} d_{I}},
$$

where $x_{i J}$ and $x_{I J}$ are the row average and block average respectively:

$$
\begin{aligned}
& x_{i J}=\frac{1}{d_{I}} \sum_{v_{j} \in V_{I}} x_{i j} \\
& x_{I J}=\frac{1}{N_{I} d_{I}} \sum_{x_{i} \in C_{I}, v_{j} \in V_{I}} x_{i j}
\end{aligned}
$$

The lower is the $H$ score, the more similar are the rise and fall patterns of the expression values of different objects. On average the clusters produced by HARP contain more genes but fewer time points. They also have a slightly better average squared residue score to size (number of genes multiplied by number of time points) ratio. Figure 1 shows the clusters with the best scores. According to the results, HARP was able to identify clusters with diverse sizes and dimensionalities. It also successfully grouped together 


\begin{tabular}{lll}
\hline Algorithm & Cheng and Church & HARP \\
\hline Avg. no. of genes & 167 & 243 \\
Avg. no. of time points & 12 & 10 \\
Avg. $H$ score & 204 & 203 \\
Avg. score to size ratio & 0.10 & 0.08 \\
\hline
\end{tabular}

Table 2. Comparison of the clusters identified by HARP and those reported Cheng and Church 2000 from the yeast data.

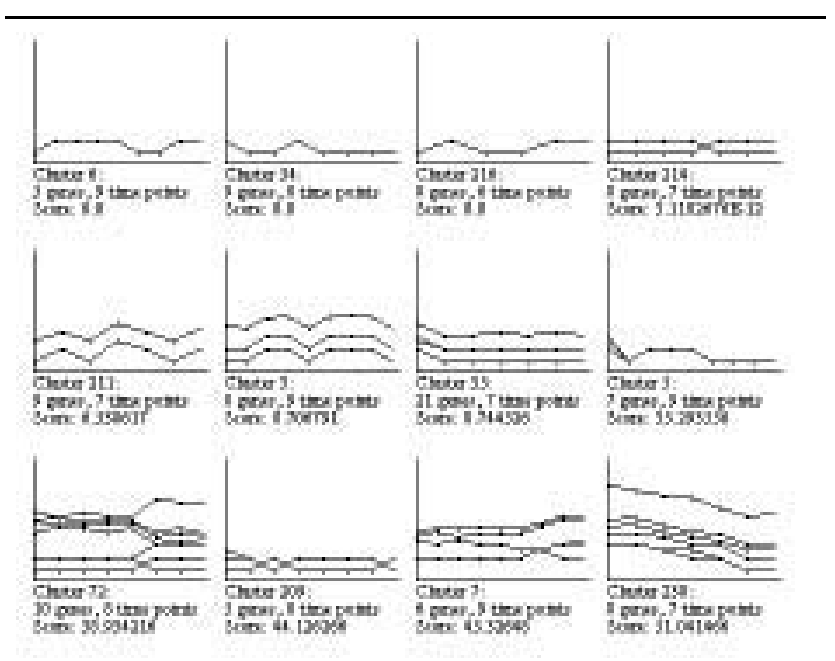

Figure 1. The clusters identified by HARP from the yeast data with the best mean squared residue scores.

genes with similar expression patterns but in opposite directions. The average size of the clusters suggests that a significant number of genes were assigned to multiple clusters with matched signatures.

We evaluated the biological significance of the clusters by a phenotypic categorization of mRNAs that are regulated with the cell cycle (http: / /yscdp. stanford.edu/ yeast_cell_cycle/functional_categories.html).

Some clusters were found to contain a significant amount of genes from related categories. One such clusters is shown in Table 3, which contains many categorized genes in the late G1 phase, with functions ranging from budding, cell cycle regulation, nuclear segregation to DNA replication and repair.

\section{Discussions}

The results show that HARP can identify statistically and biologically meaningful clusters without relying on user parameters whose proper values are hard to determine. It can thus be used to automatically identify some interesting clusters from a large number of datasets for later, more laborintensive analysis.
Category: genes

Budding, directional growth: YDR507C

Cell cycle regulators: YPL256C, YJL187C

Chromosome, nuclear segregation: YMR076C,

YDL003W, YKL042W, YMR078C

DNA repair and recombination: YLR383W, YDR097C

DNA replication: YOR074C, YLR103C, YAR007C, YNL312W, YDL164C, YBR088C

Table 3. One of the clusters identified by HARP from the yeast data that contains a significant amount of genes from related categories (all in late G1 phase).

The object assignment extension discovered some interesting non-disjoint clusters from the yeast dataset, but in general some important clusters could be missed if their structures are not captured by some disjoint clusters before object assignment. We propose two future extensions of HARP for identifying these clusters: to allow each cluster to be merged with multiple clusters, and to produce disjoint clusters on different small data samples, and then reassign other objects to the clusters. Both approaches allow the discovery of more projected structures.

A well-known weakness of hierarchical clustering algorithms is the deterministic property: once an object is assigned to a cluster, it cannot be reassigned to another. The object reassignment performed at the end of clustering helps redistribute each object to the most similar cluster, but it is unable to correct wrong merges during the early stage of clustering. We have attempted to perform an object reassignment at the end of each threshold loosening step, but no significant accuracy improvements were observed, and the clustering process was severely prolonged. We will try to integrate the threshold loosening mechanism into other more efficient and non-deterministic clustering methods.

\section{Conclusion}

In this paper, we analyzed the major challenges of the projected clustering problem, and suggested some potential weaknesses of some existing projected clustering algorithms. Based on the analysis, we proposed a new projected clustering algorithm HARP that does not rely on user inputs in determining the relevant dimensions of clusters, which makes it practical for applications where correct parameter values are hard to obtain. HARP makes use of the relevance index and dynamic threshold loosening to dynamically adjust the merging requirements of clusters according to the current clustering status. It also allows users to input some available domain knowledge, and it can be extended to perform pattern-based clustering and produce non-disjoint clusters by adaptive mean centering and post-clustering object assignment respectively. The experimental results on 
real microarray datasets show that HARP works well in situations where object similarity is based on either distance or expression pattern, and where disjoint or non-disjoint clusters are required. The clusters identified are both statistically and biologically meaningful.

\section{Acknowledgement}

The research of DWC is supported by a grant from Research Grant Council, Hong Kong. (RGC code: HKU 7141/03E). KHC is supported in part by NIH grant K25 HG02378 from the National Human Genome Research Institute and NSF grant DBI-0135442.

\section{References}

[1] C. C. Aggarwal, C. Procopiuc, J. L. Wolf, P. S. Yu, and J. S. Park. Fast algorithms for projected clustering. In $A C M$ SIGMOD International Conference on Management of Data, 1999.

[2] C. C. Aggarwal and P. S. Yu. Finding generalized projected clusters in high dimensional spaces. In ACM SIGMOD International Conference on Management of Data, 2000.

[3] R. Agrawal, J. Gehrke, D. Gunopulos, and P. Raghavan. Automatic subspace clustering of high dimensional data for data mining applications. In ACM SIGMOD International Conference on Management of Data, 1998.

[4] A. A. Alizadeh, M. B. Eisen, R. E. Davis, C. Ma, I. S. Lossos, A. Rosenwald, J. C. Boldrick, H. Sabet, T. Tran, X. Yu, J. I. Powell, L. Yang, G. E. Marti, T. Moore, J. Hudson, L. Lu, D. B. Lewis, R. Tibshirani, G. Sherlock, W. C. Chan, T. C. Greiner, D. D.Weisenburger, J. O. Armitage, R. Warnke, R. Levy, W. Wilson, M. R. Grever, J. C. Byrd, D. Botstein, P. O. Brown, and L. M. Staudt. Distinct types of diffuse large B-cell lymphoma identified by gene expression profiling. Nature, 403:503-511, 2000.

[5] U. Alon, N. Barkai, D. A. Notterman, K. Gish, S. Ybarra, D. Mack, and A. J. Levine. Broad patterns of gene expression revealed by clustering analysis of tumor and normal colon tissues probed by oligonucleotide arrays. Proc. Natl. Acad. Sci. USA, 96:6745-6750, 1999.

[6] Y. Cheng and G. M. Church. Biclustering of expression data. In Proceedings of the 8th International Conference on Intelligent Systems for Molecular Biology, 2000.

[7] R. J. Cho, M. J. Campbell, E. A. Winzeler, L. Steinmetz, A. Conway, L. Wodicka, T. G. Wolfsberg, A. E. Gabrielian, D. Landsman, D. J. Lockhart, and R. W. Davis. A genomewide transcriptional analysis of the mitotic cell cycle. Molecular Cell, 2:65-73, 1998.

[8] D. Dembl and P. Kastner. Fuzzy C-means method for clustering microarray data. BioInformatics, 19(8):973-980, 2003.

[9] M. B. Eisen, P. T. Spellman, P. O. Brown, and D. Botstein. Cluster analysis and display of genome-wide expression patterns. Proc. Natl. Acad. Sci. USA, 95:14863-14868, 1998.

[10] D. Eppstein. Fast hierarchical clustering and other applications of dynamic closest pairs. In SODA: ACM-SIAM Symposium on Discrete Algorithms, 1998.

[11] A. P. Gasch, P. T. Spellman, C. M. Kao, O. Carmel-Harel, M. B. Eisen, G. Storz, D. Botstein, and P. O. Brown. Genomic expression programs in the response of yeast cells to environmental changes. Molecular Biology of the Cell, 11:4241-4257, 2000.

[12] T. R. Golub, D. K. Slonim, P. Tamayo, C. Huard, M. Gaasenbeek, J. P. Mesirov, H. Coller, M. L. Loh, J. R. Downing, M. A. Caligiuri, C. D. Bloomfield, and E. S. Lander. Molecular classification of cancer: Class discovery and class prediction by gene expression monitoring. Science, 286(5439):531-537, 1999.

[13] J. Herrero, A. Valencia, and J. Dopazo. A hierarchical unsupervised growing neural network for clustering gene expression patterns. BioInformatics, 17(2):126-136, 2001.

[14] V. R. Iyer, M. B. Eisen, D. T. Ross, G. Schuler, T. Moore, J. C. F. Lee, J. M. Trent, L. M. Staudt, J. H. Jr., M. S. Boguski, D. Lashkari, D. Shalon, D. Botstein, and P. O. Brown. The transcriptional program in the response of human fibroblasts to serum. Science, 283:83-87, 1999.

[15] Y. Kluger, R. Basri, J. T. Chang, and M. Gerstein. Spectral biclustering of microarray cancer data: Co-clustering genes and conditions. Genome Research, 13(4):703-716, 2003.

[16] L. Lazzeroni and A. Owen. Plaid models for gene expression data. Statistica Sinica, 12:61-86, 2002.

[17] A. V. Lukashin and R. Fuchs. Analysis of temporal gene expression profiles: Clustering by simulated annealing and determining the optimal number of clusters. Bioinformatics, 17(5):405-414, 2001.

[18] D. Matei, T. G. Graeber, R. L. Baldwin, B. Y. Karlan, J. Rao, and D. D. Chang. Gene expression in epithelial ovarian carcinoma. Oncogene, 21:6289-6298, 2002.

[19] R. T. Ng and J. Han. Efficient and effective clustering methods for spatial data mining. In 20th International Conference on Very Large Data Bases, September 12-15, 1994, Santiago, Chile proceedings, 1994.

[20] S. L. Pomeroy, P. Tamayo, M. Gaasenbeek, L. M. Sturla, M. Angelo, M. E. McLaughlin, J. Y. H. Kim, L. C. Goumnerovak, P. M. Black, C. Lau, J. C. Allen, D. Zagzag, J. M. Olson, T. Curran, C. Wetmore, J. A. Biegel, T. Poggio, S. Mukherjee, R. Rifkin, A. Califano, G. Stolovitzky, D. N. Louis, J. P. Mesirov, E. S. Lander, and T. R. Golub. Prediction of central nervous system embryonal tumour outcome based on gene expression. Nature, 415:436-442, 2002.

[21] C. M. Procopiuc, M. Jones, P. K. Agarwal, and T. M. Murali. A monte carlo algorithm for fast projective clustering. In ACM SIGMOD International Conference on Management of Data, 2002.

[22] F. D. Smet, J. Mathys, K. Marchal, G. Thijs, B. D. Moor, and Y. Moreau. Adaptive quality-based clustering of gene expression profiles. Bioinformatics, 18(5):735-746, 2002.

[23] S. Tavazoie, J. D. Hughes, M. J. Campbell, R. J. Cho, and G. M. Church. Systematic determination of genetic network architecture. Nature Genetics, 22:281-285, 1999.

[24] J. Yang, H. Wang, W. Wang, and P. Yu. Enhanced biclustering on expression data. In Proceedings of the IEEE Third Symposium on Bioinformatics and Bioengineering, 2003.

[25] K. Y. L. Yip. HARP: A practical projected clustering algorithm for mining gene expression data. Master's thesis, The University of Hong Kong, Pokfulam Road, Hong Kong, 2004. 